\title{
BMJ Global Health Potential impact of 5 years of ivermectin mass drug administration on malaria outcomes in high burden countries
}

\author{
Achla Marathe, ${ }^{1,2}$ Ruoding Shi, ${ }^{2}$ Ana Mendez-Lopez, ${ }^{3,4}$ Zhihao Hu, ${ }^{5}$ Bryan Lewis, ${ }^{1}$ \\ Regina Rabinovich, ${ }^{4}$ Carlos J Chaccour (id , ${ }^{4,6}$ Cassidy Rist (i) ${ }^{7}$
}

To cite: Marathe A, Shi $R$, Mendez-Lopez A, et al. Potential impact of 5 years of ivermectin mass drug administration on malaria outcomes in high burden countries. BMJ Global Health 2021;6:e006424. doi:10.1136/ bmjgh-2021-006424

Handling editor Alberto $L$ Garcia-Basteiro

- Additional supplemental material is published online only. To view, please visit the journal online (http://dx.doi.org/10. 1136/bmjgh-2021-006424)

$\mathrm{RS}, \mathrm{AM}-\mathrm{L}, \mathrm{CJC}$ and $\mathrm{CR}$ contributed equally.

Received 26 May 2021 Accepted 18 0ctober 2021

Check for updates

(C) Author(s) (or their employer(s)) 2021. Re-use permitted under CC BY-NC. No commercial re-use. See rights and permissions. Published by BMJ.

For numbered affiliations see end of article.

Correspondence to

Dr Cassidy Rist; crist6@vt.edu

\section{ABSTRACT}

Introduction The global progress against malaria has slowed significantly since 2017. As the current malaria control tools seem insufficient to get the trend back on track, several clinical trials are investigating ivermectin mass drug administration (iMDA) as a potential additional vector control tool; however, the health impacts and costeffectiveness of this new strategy remain unclear. Methods We developed an analytical tool based on a full factorial experimental design to assess the potential impact of IMDA in nine high burden sub-Saharan African countries. The simulated iMDA regimen was assumed to be delivered monthly to the targeted population for 3 months each year from 2023 to 2027. A broad set of parameters of ivermectin efficacy, uptake levels and global intervention scenarios were used to predict averted malaria cases and deaths. We then explored the potential averted treatment costs, expected implementation costs and cost-effectiveness ratios under different scenarios. Results In the scenario where coverage of malaria interventions was maintained at 2018 levels, we found that IMDA in these nine countries has the potential to reverse the predicted growth of malaria burden by averting $20-50$ million cases and 36 000-90 000 deaths with an assumed efficacy of $20 \%$. If iMDA has an efficacy of $40 \%$, we predict between 40-99 million cases and 73 000-179 000 deaths will be averted with an estimated net cost per case averted between US\$2 and US\$7, and net cost per death averted between US\$1460 and US\$4374.

Conclusion This study measures the potential of IMDA to reverse the increasing number of malaria cases for several sub-Saharan African countries. With additional efficacy information from ongoing clinical trials and country-level modifications, our analytical tool can help determine the appropriate uptake strategies of iMDA by calculating potential marginal gains and costs under different scenarios.

\section{INTRODUCTION}

As a longstanding health problem in human history, malaria remains endemic in most WHO regions and puts millions of people at risk of infection and death each year. ${ }^{12}$ Despite being preventable and treatable, malaria disproportionately threatens vulnerable populations in many countries. The Global Technical Strategy

\section{Key questions}

What is already known?

- The global progress in malaria elimination is unlikeIy to meet the Global Technical Strategy for Malaria 2016-2030 targets due to the challenge of residual transmission, mosquito resistance to insecticides from traditional malaria control tools and more recently from challenges introduced by COVID-19.

- Ivermectin mass drug administration (iMDA) is a potential additional tool that targets mosquitoes responsible for residual transmission, and has a different mode of action in the mosquitoes as compared with current insecticides.

- Several clinical trials for iMDA are underway, however, its potential population-level health impact and cost-effectiveness have yet to be investigated.

\section{What are the new findings?}

- The predicted health and economic benefits of iMDA in the nine study countries are most significant when (i) efficacy of iMDA is high (40\% reduction in cases), (ii) global progress on malaria is slowed or stagnant and (iii) iMDA is scaled up to the national level rapidly.

- Substantial heterogeneity in cost-effectiveness was observed across countries mainly due to the difference in malaria incidence rates.

What do the new findings imply?

- Given the current challenges in malaria control and elimination globally, adding iMDA to existing vector control interventions could have significant health impacts for several sub-Saharan African countries.

- When combined with efficacy results from ongoing clinical trials with iMDA, investigation of the economic costs and benefits of iMDA as a malaria control tool will better inform country-level implementation strategy.

- Given the relationship between malaria incidence and cost-effectiveness, we anticipate a subnational approach that allows countries to target districts with the highest malaria incidence first, will achieve the greatest health impact at the lowest net cost.

for Malaria 2016-2030 (GTS) targets a reduction of malaria cases by at least $40 \%$ in 2020 compared with 2015. ${ }^{1}$ However, as of 2017, malaria cases have stagnated, and it is anticipated 
that the 2020 GTS goals for reduction in malaria incidence and deaths will be missed by $37 \%$ and $22 \%$, respectively. ${ }^{3}$ This stagnation is due to several unique challenges. First, a lack of sustained domestic and international funding has caused the battle against malaria to lose ground in some places, especially in low-income countries in sub-Saharan Africa. ${ }^{3}$ Second, the effectiveness of several widely-used vector control tools has been weakened by mosquitoes' resistance to insecticides and mosquitoes' behaviours, leading to residual transmission. ${ }^{145}$ Finally, the recent outbreak of COVID-19 has had an overwhelming impact on the health systems of countries that suffer from malaria and may significantly restrict their ability to implement usual prevention and treatment interventions. ${ }^{6}$ These challenges call for combining existing malaria control strategies with innovative methods and implementable programmes in countries that bear the highest burden of disease. ${ }^{7}$ Current and future innovations in novel vector control tools face considerable challenges, as they must address the intrinsic limitations of current vector control tools, as well as demonstrate cost-effectiveness given the current funding deficits.

Mass drug administration with systemic insecticides is a potential vector control tool that partly addresses the limitations of indoor residual spraying and insecticidetreated nets. ${ }^{8}$ Specifically, this study focuses on ivermectin mass drug administration (iMDA), which is currently applied in several African countries as part of neglected tropical disease (NTD) control and elimination activities. iMDA has been considered by the WHO as a potential additional tool for targeting both indoor and outdoor biting mosquitoes responsible for residual malaria transmission, given its safety and feasibility of implementation. ${ }^{9-12}$ After taking ivermectin, the blood of treated humans has a measurable impact on the survival of Anopheles mosquitoes feeding on them that may last up to 28 days. This effect is dependent on the ivermectin dose used and the susceptibility of the particular species involved. ${ }^{14}$ Blood meals containing ivermectin not only increase vector mortality, but also reduce sporogony, and delay refeeding frequency. ${ }^{15}$ Beyond looking at entomological outcomes, a small-scale iMDA trial to assess impact on malaria incidence has been performed under field conditions in Burkina Faso, with positive results. ${ }^{16}$

Although several studies are investigating the safety and mosquitocidal efficacy of iMDA as a malaria vector control tool, little is known about the population-level health impacts and cost-effectiveness of this strategy due to the lack of large-scale field data. To prioritise a potential new intervention, policymakers need cost-effectiveness analyses to assess the trade-off between needed resources and the potential improvement in health outcomes. Numerous studies have estimated cost-effectiveness for existing malaria control interventions, ${ }^{17-19}$ but assessing the economic viability and potential impacts of an innovative control tool with minimal data can be challenging, making policymakers reluctant to add the new tool to existing programmes.
In response to this gap, a Phase III cluster randomised, open label, clinical trial called the Broad One Health Endectocide-based Malaria Intervention in Africa (BOHEMIA) is testing the efficacy of iMDA on malariarelated epidemiological outcomes when given to humans and when given simultaneously to humans and livestock (https://bohemiaconsortium.org/). As a preparatory analysis for the BOHEMIA trial, this study investigates potential implementation costs and impact of iMDA in humans with different assumptions of iMDA efficacy and uptake levels. The analysis is performed for select countries included in the WHO's high-burden-high-impact (HBHI) strategy, ${ }^{3}$ which was released in 2018 and identifies 11 countries in which approximately $70 \%$ of the world's malaria burden is concentrated.

The global progress towards malaria elimination mainly depends on how these countries can adopt innovative solutions that are cost-effective and fit their financial capacity, which is the main focus of this study. Based on previous literature, available data and a few assumptions, the present work provides an analytical tool to estimate the marginal benefits and costs added by iMDA, including averted cases and averted deaths from malaria under different counterfactual intervention scenarios. While insufficient field data for a new vector control intervention impede a formal cost-effectiveness analysis, this analytical tool can serve as an intermediary way to guide policymakers and help evaluate potential outcomes of different uptake strategies.

\section{METHODS \\ Patient and public involvement}

This study did not involve patient or public involvement.

\section{Study design}

This study used an experimental full factorial design to simulate the potential impact of an iMDA intervention on malaria-related health and economic outcomes for the period 2023-2027, which is the 5-year period following the expected finalisation of the BOHEMIA trial in 2022. This time period was chosen given an assumption that BOHEMIA and other trials being conducted in West Africa and Asia have positive results (ie, the iMDA is effective and does reduce malaria incidence), and provide adequate data to support a WHO policy recommendation for iMDA as a new class of complementary malaria vector control tool. Three key assumptions, some derived from previous literature, were used in the simulation work: (1) the mosquitocidal impact of iMDA is positive and significant ${ }^{13} ;(2)$ several standard malaria control interventions are likely to occur during the study period and will follow one of the global scenarios developed by GTS based on the work by Griffin, et $a l^{20}$ and (3) it is assumed that, during the 2023-2027 period, funds could be available to cover the delivery costs as well as the costs required to implement and sustain iMDA campaigns. For the estimated health benefits, we simulated the impact 
of iMDA on annual cumulative number of averted cases and deaths from 2023 to 2027; during which the iMDA regimen was assumed to be a single dose of $400 \mu \mathrm{g} / \mathrm{kg}$ using $6 \mathrm{mg}$ tablets (population average 3.3 tablets per person per round) delivered monthly to the targeted population for 3 months in each year.

\section{Study area}

The potential impact of iMDA was modelled for nine countries out of the following 11 HBHI countries: Burkina Faso, Democratic Republic of the Congo, Ghana, Mali, Mozambique, Niger, Nigeria, Uganda, United Republic of Tanzania, India and Cameroon. There are considerable variations in malaria incidence rates and deaths among these nine countries in subSaharan Africa (online supplemental file S0). The two countries excluded from this analysis, Cameroon and India, are excluded for the following reasons: Ivermectin can cause severe encephalopathy in patients with high Loa loa burden. Cameroon has a high prevalence Loa loa with clinical eye worm infections in at least half of its territory. In India, although the majority of the country's population is considered to be at risk, malaria transmission intensities are generally very low, with moderate transmission areas concentrated in only 7 out of 36 states near forest fringes. ${ }^{21}$ Given the size of India's population and low national-level incidence, simulating iMDA implementation at the national level as designed by this modelling study would include a scenario that would not be a prudent use of resources.

\section{Scenario settings}

This section describes the scenario settings for which the impact was evaluated. These scenarios assume varying levels of drug efficacy, uptake levels, proportions of severe cases and underlying global malaria intervention strategies (table 1).

\section{Efficacy and severity}

The assumed efficacy of iMDA captures the potential reduction in malaria incidence in the at-risk population. The starting at-risk population for our simulation was taken from Annex 3-F in the 2019 World Malaria Report, ${ }^{2}$ and includes people living in both low, moderate and high transmission areas. Preliminary mathematical modelling work conducted for the BOHEMIA trial sites predicted that iMDA, delivered as one dose of $400 \mu \mathrm{g} /$ $\mathrm{kg}$ a month for three consecutive months to $80 \%$ of the eligible population, was expected to reduce clinical incidence in the covered population by $40 \%-46 \%$ for 1 year.

\begin{tabular}{|c|c|c|c|}
\hline Efficacy scenarios & $\begin{array}{l}20 \% \text { efficacy } \\
\text { (very conservative estimate based on } \\
\text { minimally required criteria of the PPC } \dagger \text { ) }\end{array}$ & \multicolumn{2}{|c|}{$\begin{array}{l}40 \% \text { efficacy } \\
\text { (estimated based on BOHEMIA preliminary modelling) }\end{array}$} \\
\hline Severity & $1 \%$ & $3 \%$ & \\
\hline Uptake levels & $\begin{array}{l}\text { Conservative } \\
5 \% \text { coverage of population at risk in } 2023 \text {, with } \\
\text { increase of } 3 \% \text { coverage per year. }\end{array}$ & $\begin{array}{l}\text { Rapid } \\
5 \% \text { coverage of population } \\
\text { at risk in } 2023 \text {, with } \\
\text { increase of } 10 \% \text { coverage } \\
\text { per year. }\end{array}$ & $\begin{array}{l}\text { Nationalł } \\
\text { Start at } 5 \% \text { in } 2023 \text { and double each } \\
\text { year for first } 3 \text { years and then by } 20 \% \text { per } \\
\text { year, with goal of reaching total national } \\
\text { coverage in } 7 \text { years. }\end{array}$ \\
\hline
\end{tabular}

\begin{tabular}{|c|c|}
\hline \multirow[t]{4}{*}{$\begin{array}{l}\text { GTS§ scenarios } \\
2016-2030\end{array}$} & $\begin{array}{l}\text { Accelerate } 1 \text { (incidence: }-\mathbf{2 1} \% \text {, mortality: }-\mathbf{4 0} \% \text { ) } \\
\text { Increased coverage to } 80 \% \text { with a switch from quinine to injectable artesunate for management of severe disease and } \\
\text { seasonal malaria chemoprevention. }\end{array}$ \\
\hline & $\begin{array}{l}\text { Accelerate } 2 \text { (incidence: } \mathbf{- 5 9 \%} \text {, mortality: } \mathbf{- 7 4} \% \text { ) } \\
\text { Increased coverage to } 90 \% \text { with a switch from quinine to injectable artesunate for management of severe disease, } \\
\text { seasonal malaria chemoprevention and rectal artesunate for pre-referral treatment at the community level. }\end{array}$ \\
\hline & 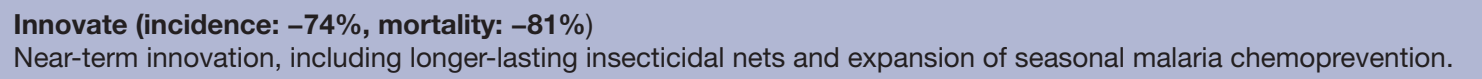 \\
\hline & $\begin{array}{l}\text { No change (incidence: } 0 \% \text {, mortality: } 0 \% \text { ) } \\
\text { Interventions remain constant over time at the } 2018 \text { levels. This scenario is used as a benchmark and is not a GTS } \\
\text { strategy. }\end{array}$ \\
\hline
\end{tabular}

Note: This table shows the scenario setting for the full factorial experimental design, including four factors. The efficacy of iMDA is defined as the assumed reduction in malaria incidence among the at-risk population. Severity refers to the percentage of severe cases among all malaria cases. Uptake levels capture the timeline and coverage of iMDA over $64 \%$ of population at risk of malaria. The projected GTS global scenarios of malaria transmission 2016-2030 were developed by Griffin, et $\mathrm{al}^{20}$ and indicated expected reduction percentages of incidence and mortality in 2030 as compared with 2015 levels.

*iMDA: ivermectin mass drug administration.

†PPC: WHO Preferred Product Characteristics.

$\mp$ Specifically, the 'National' uptake coverage rate is $5 \%$ in $2023,10 \%$ in $2024,20 \%$ in $2025,40 \%$ in $2026,60 \%$ in $2027,80 \%$ in 2028 and $100 \%$ in 2029, but this study only simulates for the 2023-2027 period.

§GTS: Global Technical Strategy for Malaria.

BOHEMIA, Broad One Health Endectocide-based Malaria Intervention in Africa. 
The preliminary modelling work was conducted by Dr Hannah C Slater (Malaria Modelling Research Group at Imperial College London) under a consulting contract with the BOHEMIA team. The modelling details are not publicly available as required by the terms of the contract, but similar modelling approaches are described in Slater $e t a .^{12}$. The eligible population excluded children under $15 \mathrm{~kg}$, pregnant women and the severely ill, which collectively account for approximately $20 \%$ of the population at risk, so the target population to cover was $64 \%$ of population at risk ( $80 \%$ of the eligible $80 \%$ ). The current analysis assumed two levels of intervention efficacy: the minimum efficacy was a $20 \%$ reduction in incidence of all clinical malaria episodes as a minimally required criterion in the WHO Preferred Product Characteristics (PPC), and the maximum efficacy was $40 \%$, the most likely estimate based on preliminary modelling. According to World Malaria Report 2019, ${ }^{2}$ between $1 \%$ and $3 \%$ of uncomplicated cases were expected to move to the severe stage, so two proportions of severe cases were used in the simulation as a sensitivity analysis.

\section{Uptake scenarios}

The uptake coverage rate was measured as the percent of the target population (ie, $64 \%$ of at-risk population) that would receive ivermectin in a given year, with the assumption that the drug would be delivered evenly regardless of differences in incidence rates across regions within a country. Three hypothetical uptake scenarios were used: 'Conservative', 'Rapid' and 'National'. In the 'Conservative' scenario, the uptake strategy was specified as targeting a random sample of districts per country encompassing at least $5 \%$ of the target population in the starting year of 2023. Then, the proportion of uptake would increase every year by $3 \%$, by which in 2027 , uptake would equal $17 \%$ of the target population in each selected country. The 'Rapid' uptake level assumed a starting coverage rate of $5 \%$ in 2023 , then increasing by an increment of $10 \%$ each year until 2027, reaching a total of $45 \%$ of the target population. The most ambitious scenario was 'National', in which the iMDA coverage rate would start at $5 \%$ of the target population in 2023 and double each year for the first 3 years and then by $20 \%$ per year afterwards, with a goal to reach the entire target population nationwide in 7 years, or $60 \%$ of the target population at the end of 2027.

\section{GTS intervention scenarios}

The GTS intervention scenarios were built on an individual-based simulation model conducted by Griffin et $a l^{20}$ so we refer to the intervention scenarios as Griffin scenarios in the following discussion. Griffin et al described the potential changes in malaria transmission around the world for 2016-2030 in five scenarios based on different uptake levels of current preventive or treatment measures as well as the availability of innovative options. The scenarios applied in this study were 'Accelerate 1', 'Accelerate 2', 'Innovate', 'Sustain' and one additional scenario not described by Griffin et al that we have labelled 'no change'. In their original work, the last scenario is 'reverse', which means the coverage of interventions declines to levels reported in 2006-2008. This study replaces the 'reverse' scenario with 'no change' as a baseline category, which assumes interventions remain constant over time at the 2018 level. All scenarios are briefly described in table 1 .

\section{Calculations and data sources}

To estimate the potential health benefits of this intervention, yearly malaria incidence rates and cumulative averted cases and deaths were modelled for the nine countries from 2023 to 2027 in the following manner: (1) We estimated the target population over time. This was $64 \%$ of each country's 2018 population at risk adjusted for population growth using country population growth rates from the World Bank's World Development Indicators 2018 database. $^{22}$ (2) Starting from the baseline without iMDA, we predicted the yearly rate of change of the malaria incidence based on 2015 incidence rate and the expected targets by 2030 under each Griffin scenario, ${ }^{20}$ assuming the total change was equally distributed across the 15 years. (3) Then, the baseline malaria cases $\left(M^{\text {base }}\right)$ for country $c$ at year $t$ without iMDA were predicted using 2018 actual cases $\left(M_{2018, c}\right)$, population growth rates $\left(p r_{c}\right)$ in country $c$, and the yearly rate of change (roc) derived in step (2) as follows,

$$
M_{t, c}^{\text {base }}=M_{2018, c}\left[\left(1+p r_{c}\right) \times r o c\right]^{t-2018}(1)
$$

The baseline scenario served as a counterfactual against which the impact of the iMDA intervention was assessed. Building on the baseline, hypothetical cases under intervention were modelled for every country as follows

$$
\mathbf{M}_{t, c}^{i M D A}=\left\{\begin{array}{l}
M_{2018, c}\left[\left(1+p r_{c}\right) \times r o c\right]^{t-2018}, \text { if } 2018<t<2023 \\
M_{2018, c}\left[\left(1+p r_{c}\right) \times r o c\right]^{t-2018} \times\left(1-E \times U_{t}\right), \text { if } 2023 \leq t \leq 2027
\end{array}\right.
$$

where $U_{t}$ is the uptake coverage rate at year $t$ described

in table 1 , and $E$ is an assumed efficacy level $(20 \%$ or

$40 \%)$ of ivermectin. The baseline yearly malaria deaths, $D_{t, c}^{\text {base }}$, were predicted in a similar way as steps (2) and (3) by replacing incidence rate and cases with mortality rate and deaths, respectively. The numbers of averted cases $\quad\left(M_{t, c}^{\text {averted }}=M_{t, c}^{\text {base }}-M_{t, c}^{i M D A}\right)$ and averted deaths $\left(D_{t, c}^{\text {averted }}=D_{t, c}^{\text {base }}-D_{t, c}^{i M D A}\right)$ were defined as the reduction of simulated cases and deaths after applying ivermectin compared with the baseline. The data on population at risk and malaria cases and deaths were collected from the WHO's World Malaria Report 2019. ${ }^{2}$

To assess the economic impact from the health provider's perspective, we simulated the averted treatment costs associated with the number of averted cases between 2023 and 2027 attributable to the ivermectin intervention. Regardless of public or private provider, the averted 
cost reflects the total averted healthcare expenditure on treating malaria including out-of-pocket spending. The treatment cost measures were specified as the median provider economic cost of treating an episode of malaria from the systematic review by Conteh, et $a l,^{23}$ which reported the treatment cost of an uncomplicated episode as US\$9.31 and that of a severe episode as US\$89.93 in 2018. From 2023 to 2027, the averted treatment cost $\left(C^{\text {averted }}\right)$ due to iMDA for country $c$ at year $t$ given a proportion of severe episodes $(s)$ was

$C_{t, c}^{\text {averted }}=30.26 \times M_{t, c}^{a \text { averted }} \times s+5.84 \times M_{t, c}^{\text {averted }} \times(1-s) .(3)$

The total cost of scaling up the intervention to the eligible population (ie, $64 \%$ of the country's at-risk population $P$ ) was estimated from the project implementer's perspective, including the cost of the drug and the cost of distribution in response to different uptake levels of iMDA. Based on BOHEMIA final dosage form and the estimated delivery costs for NTD programmes in African countries, ${ }^{24}$ we assumed a conservative purchasing cost of US $\$ 0.15$ per $6 \mathrm{mg}$ tablet and a distribution cost of US $\$ 0.46$ per person per round. There are three rounds in each year, delivering a mean of 3.3 tablets per person in each round. For $2023 \leq t \leq 2027$, the implementation cost was modelled as

$C_{t, c}^{i M D A}=64 \% \times P_{2023, c}\left(1+p r_{c}\right)^{t-2023} \times U_{t} \times 3 \times[0.46+0.15 \times 3.3] .(4)$

In sum, the full-factorial design consisted of 9 countries, 3 uptake levels, 2 ivermectin efficacy levels, 5 scenarios and 2 severity levels of malaria cases. A total of $540(=9 \times 3 \times 2 \times 5 \times 2)$ experiments were simulated using statistical data processing applications in $\mathrm{R}$. We adjusted all costs to 2020 US\$ using the annual inflation rate of Consumer Price Index from the US Bureau of Labor Statistics.

\section{Outcome variables}

Six outcome metrics were constructed: (i) malaria incidence per 1000 population at risk, (ii) averted cases, (iii) averted deaths, (iv) implementation cost of scaling up, (v) cumulative averted treatment cost and (vi) average cost-effectiveness ratios (CERs). Malaria incidence rates were predicted for the 2023-2027 period under different intervention scenarios. The net cost of iMDA was modelled as the scaling-up cost minus averted treatment cost attributable to iMDA. In each country, the average CERs for cost per averted case $\left(C E R_{c}^{\text {case }}\right)$ and cost per averted death $\left(C E R_{c}^{\text {death }}\right)$ were calculated by dividing the cumulative net cost over cumulative averted cases and deaths from 2023 to 2027, respectively:

$$
\begin{aligned}
& C E R_{c}^{\text {case }}=\frac{\sum_{t=2023}^{2027}\left(C_{t, c}^{i M D A}-C_{t, c}^{\text {averted }}\right)}{\sum_{t=2023}^{2027} M_{t, c}^{\text {averted }}}(5) \\
& C E R_{c}^{\text {death }}=\frac{\sum_{t=2023}^{2027}\left(C_{t, c}^{i M D A}-C_{t, c}^{\text {averted }}\right)}{\sum_{t=2023}^{2027} D_{t, c}^{\text {averted }}}(6)
\end{aligned}
$$

Along with other impact estimates, these two ratios are of particular interest because they identify the scenarios that yield the greatest reduction of malaria burden for the least resources and help to guide uptake decisions.

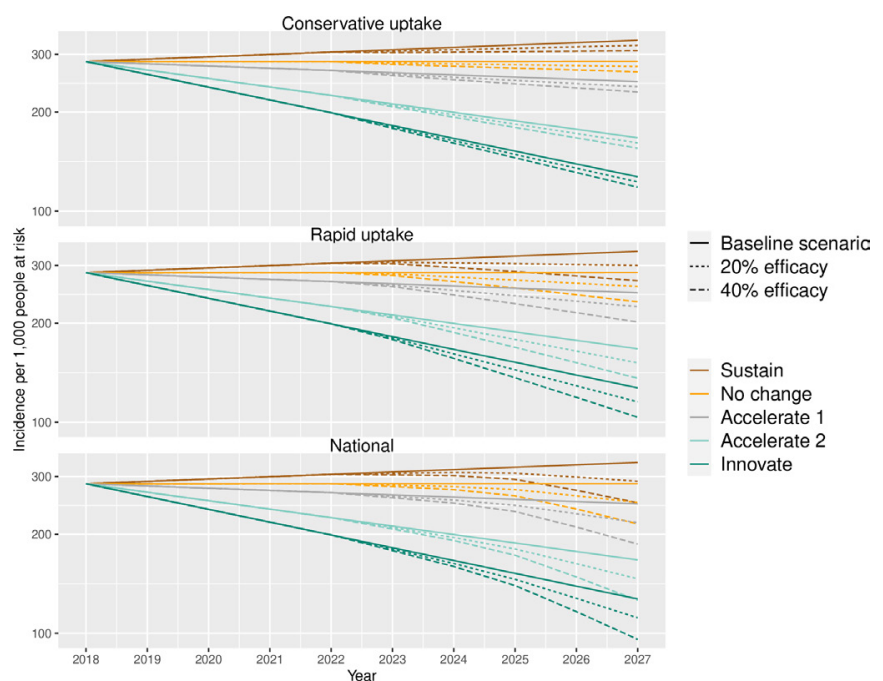

Figure 1 Simulated malaria incidence in all nine countries for different Griffin scenarios and uptake levels.

\section{RESULTS}

\section{Aggregated results across all countries}

Health impact

Figure 1 illustrates the estimated impact of iMDA on malaria incidence for the nine HBHI countries as a whole. Cumulative averted cases from 2023 to 2027 are reported in figure 2A. With a 'Conservative' uptake level (ie, starting at $5 \%$ and increasing by $3 \%$ each year), the impact of iMDA on national malaria incidence is relatively small, especially when the scenarios are 'Accelerate 2' or 'Innovate'. The largest impact is observed in the 'Sustain' scenario with an efficacy of $40 \%$, but it cannot reverse the predicted growth of malaria burden, increasing from 285.1 to 307.6 cases per 1000 population at risk by 2027 . Compared with the baseline, the cumulative averted cases in this scenario are 46 million (figure 2A).

The 'Rapid' uptake of iMDA with an efficacy of $40 \%$ is expected to reverse the growth of malaria incidence. Even under the worst 'Sustain' scenario, the intervention generates a downward trend in malaria incidence after initiation in 2023. Malaria incidence under the 'Sustain' scenario falls below the 'No change' baseline scenario after 2025, and a similar overlapping is observed between 'No change' and 'Accelerate 1' scenarios. Even though the projected malaria incidence is not lower than 2018 levels when the efficacy is $20 \%$ under 'Sustain' scenario, cumulative averted cases are notable, ranging from 23 million in the 'Innovative' scenario to 52 million in the 'Sustain' scenario (figure 2A).

The impact of iMDA is most considerable if the intervention can be quickly expanded nationwide in 7 years. The lower panel of figure 1 shows that even for 'Sustain' and 'No change' scenarios (ie, the least favourable for malaria control), iMDA leads to a downward-sloping curve from 2024. Take the 'Sustain' scenario as an example. With an efficacy of $20 \%$, iMDA can bring the growing malaria burden back to the 2018 level within 10 years and avert 57 million cases from 2023 to 2027 (figure 2A). With 40\% 
A.

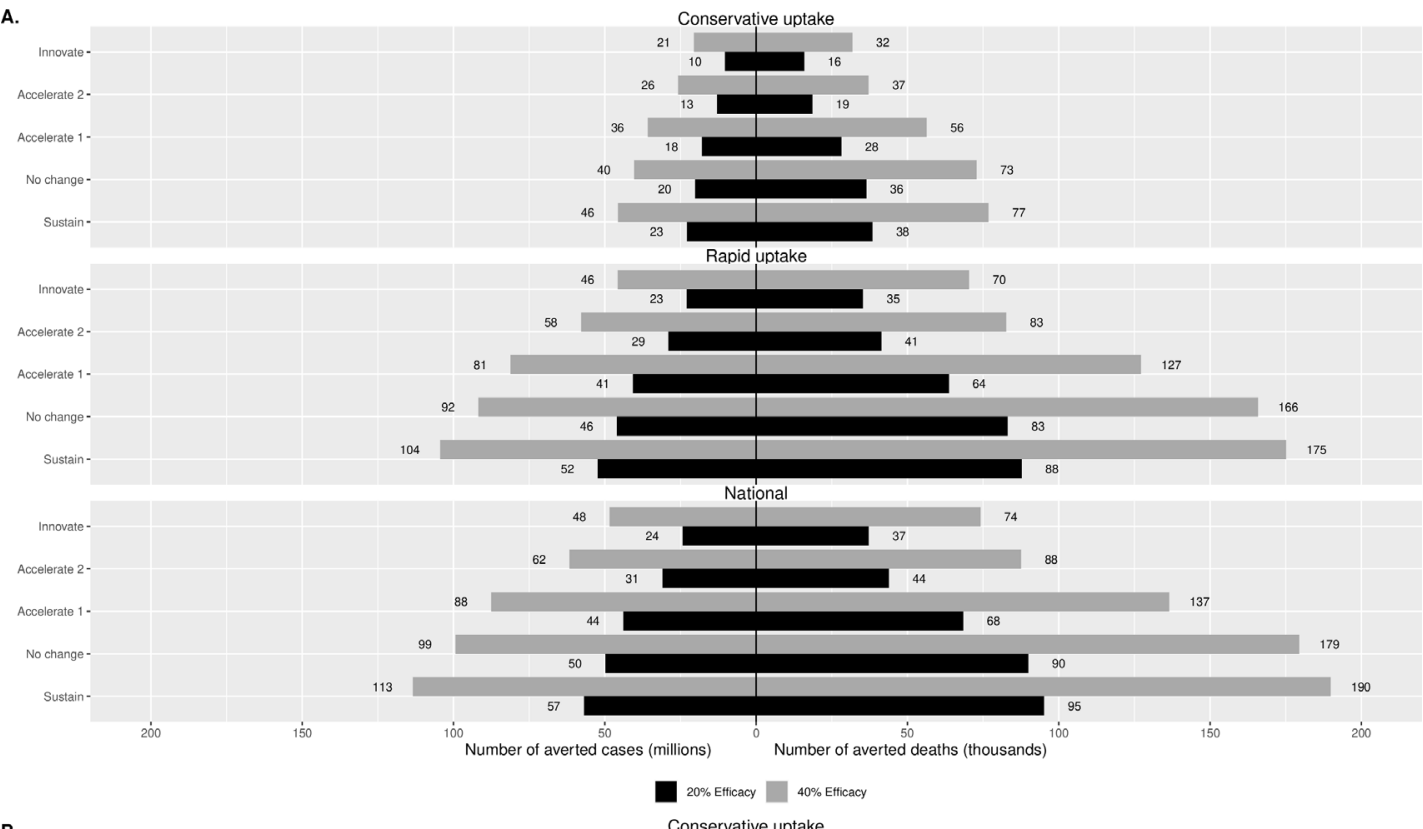

B.

Conservative uptake

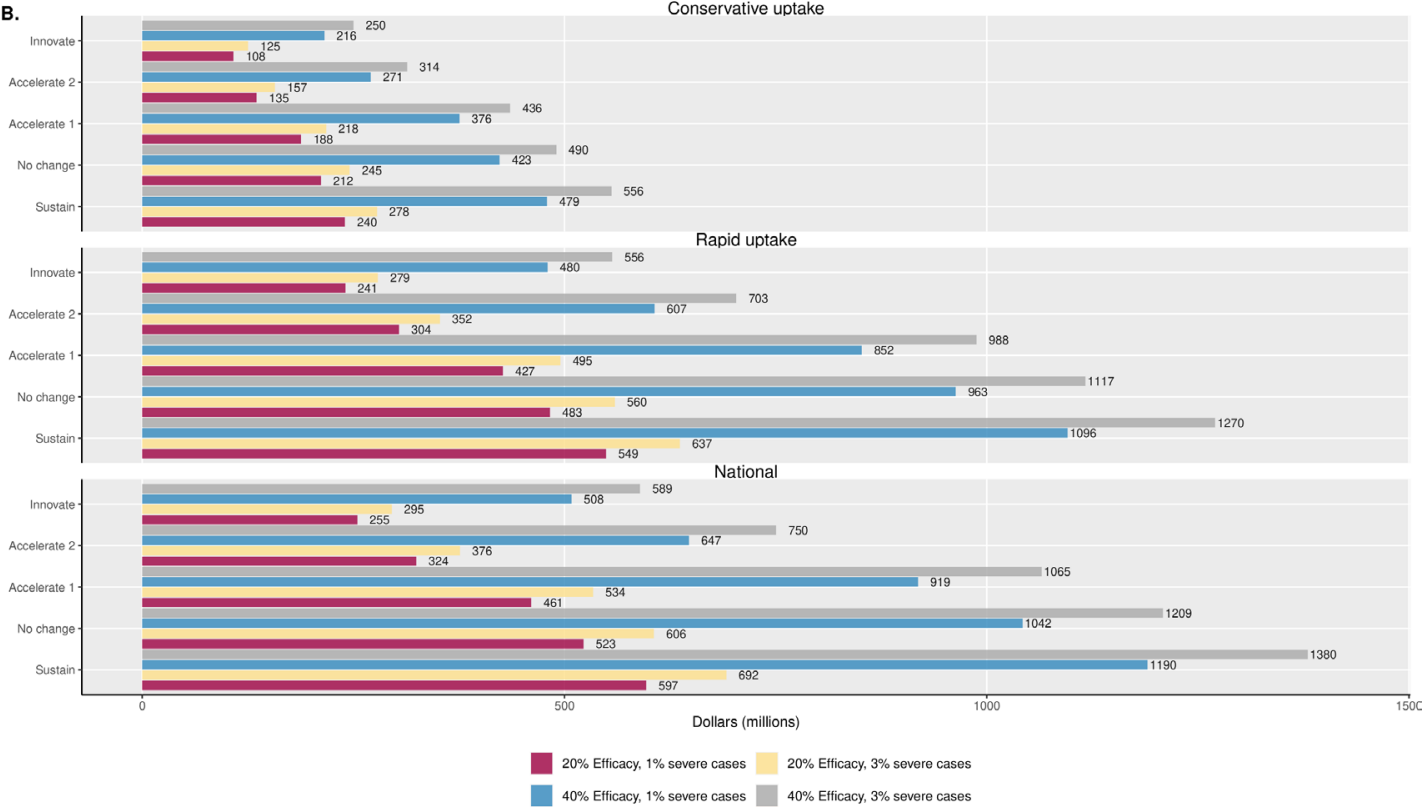

Figure 2 Simulated impacts of ivermectin mass drug administration in all nine countries for different Griffin scenario and uptake levels. (A) Cumulative averted cases and deaths and (B) cumulative averted financial costs.

efficacy, iMDA is estimated to avert 113 million cases and reduce malaria incidence to 249.5 per 1000 population at risk by the end of 2027 , which is lower than the baseline incidence in the 'No change' scenario. However, the impact tends to be smaller under the 'Innovate' scenario compared with other scenarios, which includes longerlasting insecticidal nets and expanded seasonal malaria chemoprevention for children aged 3 months up to 10 years. ${ }^{20}$ Under the 'Innovate' scenario, the estimated cumulative averted cases are between 24 million (efficacy $=20 \%$ ) to 48 million (efficacy $=40 \%$ ).

In addition to cumulative averted cases, figure 2A presents cumulative averted deaths due to three uptake levels of ivermectin, compared with the baseline. When the global malaria scenario is 'Sustain' with an efficacy of
20\%, a 'Conservative' iMDA uptake can avert a total of 38 000 deaths in 5 years and the numbers of averted deaths are 88000 and 95000 under 'Rapid' and 'National' uptake scenarios, respectively. Under the 'No change' global progress with an efficacy of $20 \%$, the number of averted deaths range from 36000 to 90000 . Consistent with the results of averted cases, the numbers are smaller when the global progress is 'Innovate' compared with the 'Sustain' and 'No change' cases.

\section{Economic impact and CER}

Figure 2B shows the cumulative averted financial costs due to iMDA under three uptake levels. Since the treatment cost of a severe episode is about 10 times higher than that of an uncomplicated episode, the averted costs 
Table 2 An overall summary of gross/net cost and cost-effectiveness of iMDA* for all nine countries

\begin{tabular}{|c|c|c|c|c|c|c|}
\hline Uptake & Efficacy & Griffin scenario & $\begin{array}{l}\text { Scale-up cost† } \\
\text { (million US\$) }\end{array}$ & $\begin{array}{l}\text { Net cost } \\
\text { (million US\$) }\end{array}$ & $\begin{array}{l}\text { CER§ for averted } \\
\text { cases (US\$) }\end{array}$ & $\begin{array}{l}\text { CER§ for averted } \\
\text { deaths (US\$) }\end{array}$ \\
\hline \multirow[t]{6}{*}{ Conservative } & $40 \%$ & Sustain & 667.8 & 112.1 & 2 & 1460 \\
\hline & & No change & 667.8 & 177.4 & 4 & 2435 \\
\hline & & Accelerate 1 & 667.8 & 232.3 & 6 & 4123 \\
\hline & $20 \%$ & Sustain & 667.8 & 389.7 & 17 & 10141 \\
\hline & & No change & 667.8 & 422.3 & 21 & 11585 \\
\hline & & Accelerate 1 & 667.8 & 449.8 & 25 & 15954 \\
\hline \multirow[t]{6}{*}{ Rapid } & $40 \%$ & Sustain & 1528.8 & 258.4 & 2 & 1475 \\
\hline & & No change & 1528.8 & 412.0 & 4 & 2484 \\
\hline & & Accelerate 1 & 1528.8 & 540.9 & 7 & 4253 \\
\hline & $20 \%$ & Sustain & 1528.8 & 892.1 & 17 & 10164 \\
\hline & & No change & 1528.8 & 969.1 & 21 & 11659 \\
\hline & & Accelerate 1 & 1528.8 & 1033.7 & 25 & 16217 \\
\hline \multirow[t]{6}{*}{ National } & $40 \%$ & Sustain & 1662.3 & 282.2 & 2 & 1487 \\
\hline & & No change & 1662.3 & 453.7 & 5 & 5057 \\
\hline & & Accelerate 1 & 1662.3 & 597.2 & 7 & 4374 \\
\hline & $20 \%$ & Sustain & 1662.3 & 970.5 & 17 & 10199 \\
\hline & & No change & 1662.3 & 1056.5 & 21 & 11742 \\
\hline & & Accelerate 1 & 1662.3 & 1128.4 & 26 & 16486 \\
\hline
\end{tabular}

All numbers are inflation-adjusted to 2020 US\$ and reflect summations from 2023 to 2027 in the nine countries where the ivermectin intervention is applied; severity is set at $3 \%$.

*iMDA: ivermectin mass drug administration.

†Scale-up cost is the cost of implementing iMDA and includes costs of drug and drug delivery.

$\ddagger$ Net cost of iMDA is the scale-up cost of iMDA minus the cumulative treatment cost averted due to iMDA.

§CER: cost-effectiveness ratio.

not only depend on the efficacy of ivermectin but also on the proportion of severe cases. Under the 'Conservative' uptake level, the cumulative averted treatment costs can range from US $\$ 108$ million at 2020 (in 'Innovative' scenario, efficacy $20 \%$ and severity $1 \%$ ) to US $\$ 556$ million (in 'Sustain' scenario, efficacy $40 \%$ and severity $3 \%)$ across all nine countries. The figure shows that the simulated averted costs are most sensitive to the assumption of efficacy but quite robust to different severity proportions.

Following a similar pattern, the cumulative averted costs under 'Rapid' uptake range from US\$241 million to US $\$ 1.27$ billion, which are more than twice of the averted costs in the 'Conservative' uptake scenario. Under 'National', the averted costs are slightly higher compared with the costs under the 'Rapid' uptake, that is, from US $\$ 255$ million up to US $\$ 1.38$ billion.

Table 2 shows the overall gross and net costs of implementing the iMDA in these nine countries for the period from 2023 to 2027, as well as CERs calculated based on averted cases and deaths. Although there are a total of 60 scenarios in the full-factorial design (online supplemental file S1), only 18 selected scenarios are presented in this table. According to a geospatial modelling estimated by WHO, ${ }^{6}$ the current COVID-19 pandemic is a top evolving risk to increase malaria cases and deaths for the year 2020 because of potential delaying or interrupting treatments and intervention programmes. Therefore, the selected scenarios of 'Sustain', 'No change' and 'Accelerate 1' are more likely to reflect the possible current paths than the scenarios of 'Accelerate 2' and 'Innovate'. As results do not change significantly with severity proportions, we use $3 \%$ severity for the main set of results. Similar results based on $1 \%$ severity can be found in online supplemental tables A-C in S1 file.

The gross intervention costs reflect the scale-up costs of purchasing and delivering ivermectin to the target population. Uptake level is the only factor that affects the gross intervention cost from 2023 to 2027, which is US $\$ 667.8$ million under 'Conservative' uptake, US\$1.53 billion under 'Rapid' uptake and US $\$ 1.66$ billion under 'National' uptake. The net intervention costs measure the costs of the intervention after accounting for the saving in treatment costs due to averted cases. Table 2 shows that the net costs of implementing iMDA can be as low as US $\$ 112.1$ million in the 'Sustain' scenario with $40 \%$ efficacy and 'Conservative' uptake, and as high as US $\$ 1.13$ billion in 'Accelerate 1' with $20 \%$ efficacy and 'National' uptake. The cost per case averted indicates that the most cost-effective scenario for iMDA occurs when this vector control tool is highly efficacious (ie, 40\%) and when the scenario for global progress on malaria is the 
least favourable (ie, 'Sustain' scenario). In this case, the lowest net cost is $\$ 2$ per case averted and $\$ 1460$ to $\$ 1487$ per death averted, depending on the uptake strategy. However, if ivermectin is less efficacious (ie, 20\%), the net cost per case averted can range between $\$ 17$ and $\$ 26$, and the net cost per death averted is between $\$ 10141$ and \$16 486 .

\section{Country-specific results}

We conducted the above analyses for each country separately. The online Supplementary Material shows each country's simulated malaria incidence (online supplemental figure A in S11 - S19 files), cumulative averted cases (online supplemental figure B in S11 - S19 files), cumulative averted deaths (online supplemental figure C in S11 - S19 files) and cumulative averted financial costs (online supplemental figure D in S11 - S19 files). A summary of net costs per case and per death averted are presented here to compare the cost-effectiveness of iMDA among countries. Full results for each country are reported in online supplemental S2-S9.

\section{Cost-effectiveness for averted cases}

The net cost per case averted under different scenarios are shown in table 3 panel A. The CERs are robust to different severity assumptions and change little between uptake levels (see table 2 for instance). Therefore, table 3 only reports the CERs calculated based on the assumption of 'Rapid' uptake and $3 \%$ of cases becoming severe.

Depending on the Griffin scenario, the net cost per case averted ranges from US\$-2 to US $\$ 141$. The negative net cost means that total averted treatment costs exceed the scale-up costs of purchasing and delivering ivermectin to the target population in a country. In general, iMDA's value added increases as the global malaria progress slows down from 'Innovate' to 'Sustain'. In other words, iMDA is more cost-effective when other malaria interventions remain at coverage levels of 2011-2013, as compared with a situation where there are innovative gains in coverage and treatment. Based on net cost per case averted, we can rank the nine countries from lowest to highest CER as follows: Burkina Faso, Mali, Niger, Democratic Republic of the Congo, Mozambique, Nigeria, Uganda, Ghana and Tanzania. The ranking is the same under different uptake levels, and under the two levels of efficacy $(20 \%$ and $40 \%$ ). Burkina Faso has the lowest CER, with a net benefit of US $\$ 2$ under 'Sustain' scenario and a breakeven in 'No change' scenario, assuming an efficacy of $40 \%$. When an efficacy of $20 \%$ is assumed, the CERs increase by US $\$ 11$ to US $\$ 23$, reaching the highest value under the 'Innovate' scenario where the net cost is US $\$ 35$ per case averted. Comparatively, iMDA in Tanzania has the highest CER, where net costs per case averted range from US\$21 to US\$65 with an assumed efficacy of $40 \%$, or US $\$ 55$ to US $\$ 141$ (more than double) when an efficacy of $20 \%$ is assumed.

\section{Cost-effectiveness for averted deaths}

Table 3 panel B shows the country-specific net costs per death averted due to iMDA. A comparison between countries suggests that iMDA in Burkina Faso, Mali and Niger are expected to have lower CERs than in other countries. For instance, if global malaria interventions reverse back to the coverage levels of 2011-2013 (ie, 'Sustain' scenario) due to the COVID-19 pandemic, the averted cost per death by an iMDA with $40 \%$ efficacy in these three countries will exceed its implementation cost. Take Burkina Faso as an example, the implementation cost of a rapid uptake in 'Sustain' scenario is US\$60.3 million, and the averted treatment cost is US\$70.3 million with 18852 averted deaths (online supplemental file S6 table B). However, if the scenario is 'Innovative', a rapid uptake of iMDA in Burkina Faso can only avert 7576 deaths and US\$30.8 million treatment costs, in whichCER death $=3,895$.

On the other hand, Tanzania, Uganda and Ghana and have relatively high CERs among study countries. Take Tanzania as an example, the net cost in the 'Sustain' scenario is higher than that in other countries, ranging from US $\$ 7505$ to US\$19 211 per death averted, depending on the level of efficacy. However, in this case, the intervention still averts 7438 to 14842 additional deaths (online supplemental file S10 table B), which is not insignificant.

\section{DISCUSSION}

The main findings highlight a few observations about the potential impact and economic viability of iMDA. First, it is important to know the current level of progress being made against malaria globally. Due to the disruption of COVID-19, the 'Sustain' and 'No change' scenarios are more likely to be reflective of the future malaria landscape,${ }^{625}$ so we focus on these two scenarios in the following discussion. Simulated malaria incidence shows that while only 'Rapid' or 'National' uptake of iMDA can tame the increasing overall malaria burden, a 'Conservative' uptake would still help slow down the increasing trends by averting malaria cases and deaths. Second, averted treatment costs are most sensitive to iMDA efficacy. Nonetheless, even if a higher efficacy level (40\%) yields lower net costs, a lower efficacy level (20\%) still contributes to reducing the global malaria burden. Largescale, randomised controlled trials of iMDA in at-risk populations are currently underway with BOHEMIA (https://bohemiaconsortium.org/) and other relevant trials. ${ }^{26-29}$ Results of these trials will better estimate efficacy of iMDA, and can be used to further refine expectations of the cost-effectiveness of iMDA strategies. ${ }^{163031}$

Even with the lowest efficacy of iMDA estimated at 20\%, the simulated results suggest that a rapid uptake of iMDA can avert a total of 46-52 million malaria cases in these countries, leading to a treatment cost savings of around US $\$ 500$ million and over 80000 deaths averted. Given the 218 million estimated total malaria cases in $2015,{ }^{25}$ 


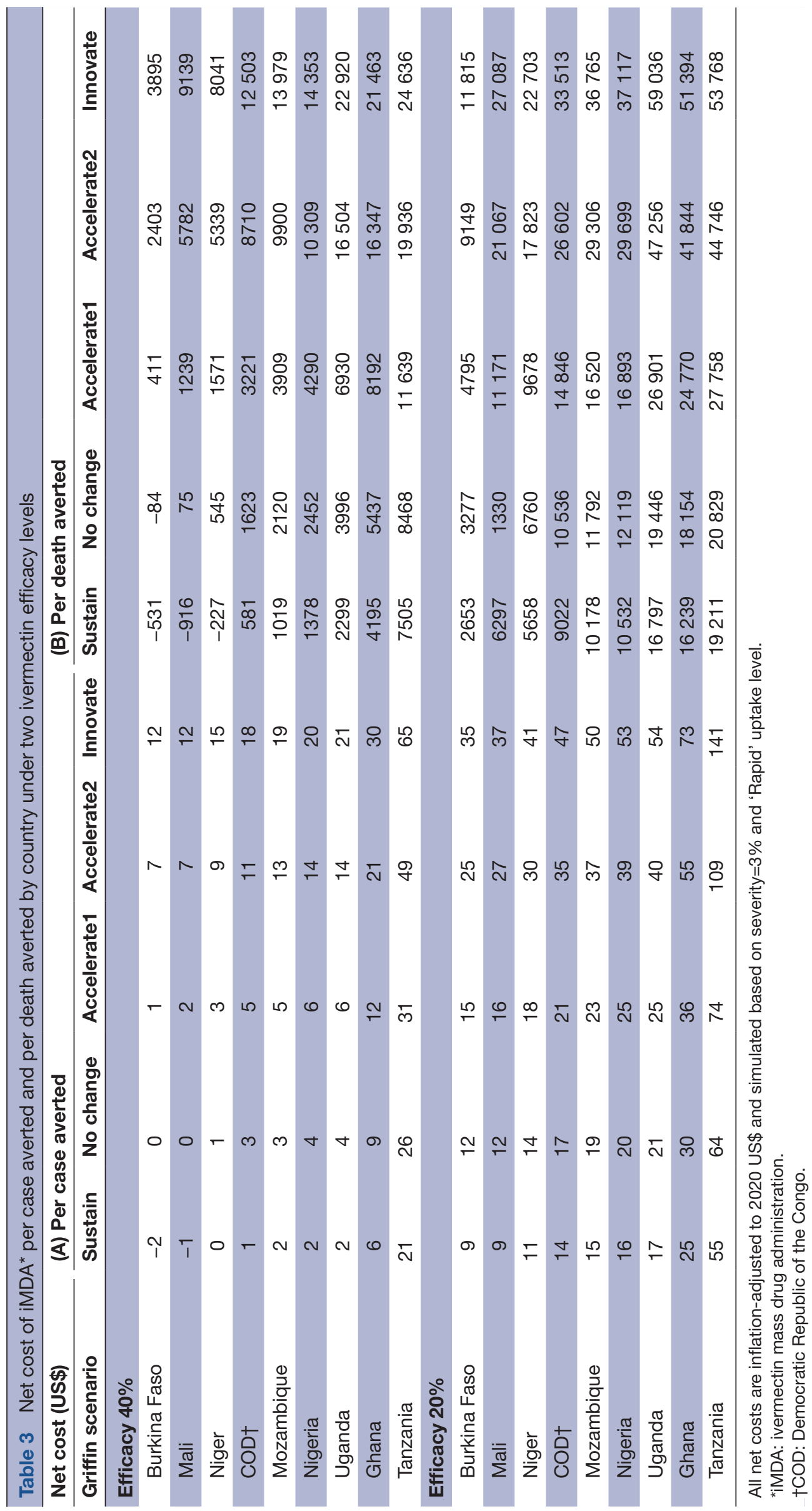


this scenario would lead to a total of $21 \%-23 \%$ reduction in world malaria cases over the 5-year period from 2023 to 2027, contributing to the GTS goal considerably. On the other hand, iMDA will widen the gap between the invested funding and the financing targets for malaria control and elimination under the GTS goal. ${ }^{32}$ This study finds that scaling up iMDA in the nine selected HBHI countries can cost a total of US $\$ 0.67$ billion under 'Conservative' uptake, US $\$ 1.53$ billion under 'Rapid' uptake and US $\$ 1.66$ billion under 'National' uptake from 2023 to 2027. From 2016 to 2018, there was an estimated US $\$ 3.5$ billion reported by the $11 \mathrm{HBHI}$ countries to fight against malaria. ${ }^{2}$ Assuming funding levels for HBHI countries remains the same, implementing this intervention under the three uptake levels will potentially increase yearly funding needs by $11.5 \%, 26.2 \%$ and $28.5 \%$, respectively. Moreover, the costs of rapid scale-up of iMDA can be relatively large for some countries and challenges the economic viability of adopting this new tool nationwide. Given this, there may be options for countries to consider that may help lower costs while targeting the highest at-risk populations at a subnational level or sharing cost with some NTD programmes. For example, several NTD programmes deliver ivermectin to people once a year in all HBHI countries, as ivermectin is effective against a number of other parasites, namely onchocerciasis and lymphatic filariasis. If their implementation period can partly overlap with iMDA for malaria, one possible way to reduce the scale-up cost and improve cost-effectiveness is to share one round of ivermectin delivery. Moreover, the collaboration with NTD programmes may reduce implementation budgets by benefiting from existing NTD programme infrastructure and human resources, as well as the population's social acceptance of iMDA given longstanding NTD interventions. However, coordination may not be easy, given differences in target populations and timing of programmes. A significant amount of discussion would need to occur at the country-level to ensure benefits would be achieved for both the NTD and malaria programmes.

The heterogeneity in country-specific results implies that there is no one-size-fits-all malaria control strategy and countries should select the most effective malaria control strategy for the least cost. The difference in simulated CERs is mainly due to different initial malaria incidence rates between countries. Holding other factors the same, iMDA is expected to avert more malaria cases in a country with a higher incidence than in a country with a lower incidence. This approach could be taken at a subnational level, where rather than scaling-up equally across the at-risk population as a whole (as was modelled in this study), countries target scale-up in districts with the highest malaria incidence first, to have the greatest health impacts for the lowest net cost. Although implementing iMDA in countries or districts at the subnational level with lower incidence is more costly, it has a potential to accelerate malaria elimination, which needs to be evaluated by future studies.

\section{Limitations}

Common to previous cost-effectiveness literature using simulation methods, this study has several limitations. First, the costs for treatment were estimated based on previous literature that provides an average cost across multiple studies instead of being collected from an existing country-specific iMDA programme, and the benefits were restricted to treatment cost savings without other social benefits, such as increased productivity from the cases averted, value of life years saved and the potential reduction in incidence of other NTDs. Nonetheless, the cost of delivery was based on evidence from iMDAs for NTDs in African countries and, thus, we expect the costs of our intervention to be fairly similar, although this may be overly optimistic. Second, our estimated scale-up costs were the lower-bounds of the actual costs because some cost items, such as personnel training costs, capital costs and management/monitoring costs, were not included in the simulation. However, due to economies of scale,$^{19}$ the unit cost of drug delivery tends to decrease as the intervention coverage increases, which may partly offset the underestimation of scale-up costs. Third, some parameters in the model were assumed to be fixed, such as the fraction of a country's eligible population and ivermectin efficacy. As a result, the model could not account for feedback and potential changes in immunity when endemicity is changed by the intervention. We acknowledge that accurate prediction is very challenging since many factors may change the parameters over time. By conducting a full-factorial design, we used a wide range of scenario settings to partly accommodate the uncertainty. Future studies may build on this approach by changing factors, for instance, including the correlation between per cent of severe cases and mortality rate, transmission setting and insecticide resistance. Fourth, with a focus on country-level health impacts, this model does not consider subnational iMDA strategies. Future studies may consider scaling up the intervention targeting to the areas within a country where it would be most effective to improve the overall cost-effectiveness of iMDA.

\section{CONCLUSIONS}

The analytical tool developed by this study will help frame investments in ivermectin trials, cost-effectiveness studies, as well as investments in innovations related to implementation in HBHI countries. The modelling framework can be expanded to account for countryspecific factors, such as level of malaria transmission, opportunities for cost-sharing with NTD programmes, distribution of the at-risk population over space and time, infrastructure and personnel available for programme implementation and financial resources. When refined with actual data and country inputs on delivery strategy and targeting, this can be an increasingly robust tool to support national decision-making. This study finds that iMDA is more cost-effective to implement in some countries (eg, Burkina Faso, Mali and Niger) than in others, 
such as Tanzania and Uganda; although when it comes to making policy decisions, country-specific evaluation of the cost-effectiveness of iMDA relative to or in conjunction with other in-country options is more relevant than simply comparing values across countries.

Most significantly, the results are sensitive to the efficacy of ivermectin and the underlying global intervention scenario that best reflects the current situation relative to progress on malaria intervention delivery and future innovations. The recent COVID-19 outbreak is likely resulting in substantial increase in morbidity and mortality from malaria in these countries ${ }^{33}$ which suggests that the 'Sustain', 'No change' and 'Accelerate 1' scenarios represent the more likely trajectories for malaria intervention delivery. Results of ongoing randomized-control trials that examine the efficacy level of iMDA and efforts to improve estimates of costs associated with iMDA implementation will be valuable to produce more accurate cost-effectiveness estimates. The BOHEMIA trial, on which this study was based, is estimated to have results by 2023, which will better inform future country-level analyses. With appropriate uptake strategies and validation of expected efficacy, we anticipate iMDA can be a good option for several sub-Saharan African countries to accelerate success in malaria control.

\author{
Author affiliations \\ ${ }^{1}$ Network Systems Science and Advanced Computing Division, Biocomplexity \\ Institute, University of Virginia, Charlottesville, Virginia, USA \\ ${ }^{2}$ Department of Public Health Sciences, University of Virginia, Charlottesville, \\ Virginia, USA \\ ${ }^{3}$ Department of Preventive Medicine, Public Health and Microbiology, Autonomous \\ University of Madrid, Madrid, Spain \\ ${ }^{4}$ Barcelona Institute for Global Health, ISGlobal, Barcelona, Spain \\ ${ }^{5}$ Department of Statistics, Virginia Tech, Blacksburg, Virginia, USA \\ ${ }^{6}$ Universitat de Navarra, Pamplona, Spain \\ ${ }^{7}$ Department of Population Health Sciences, Virginia Tech, Blacksburg, Virginia, USA
}

Contributors CJC and RR conceived of the study. Methods were developed by CJC, AM-L, AM, ZH and CR. Formal analysis was performed by AM, RS, AM-L, CJC, $\mathrm{ZH}$ and $\mathrm{BL}$. The manuscript was written by $\mathrm{AM}, \mathrm{RS}$ and $\mathrm{CR}$, with review and editing performed by all contributing authors. AM serves as the guarantor of this study.

Funding This study was funded by Unitaid (https://unitaid.org/project/massdistribution-of-a).

\section{Competing interests None declared.}

Patient consent for publication Not applicable.

Provenance and peer review Not commissioned; externally peer reviewed.

Data availability statement All data relevant to the study are included in the article or uploaded as supplementary information.

Supplemental material This content has been supplied by the author(s). It has not been vetted by BMJ Publishing Group Limited (BMJ) and may not have been peer-reviewed. Any opinions or recommendations discussed are solely those of the author(s) and are not endorsed by BMJ. BMJ disclaims all liability and responsibility arising from any reliance placed on the content. Where the content includes any translated material, BMJ does not warrant the accuracy and reliability of the translations (including but not limited to local regulations, clinical guidelines, terminology, drug names and drug dosages), and is not responsible for any error and/or omissions arising from translation and adaptation or otherwise.

Open access This is an open access article distributed in accordance with the Creative Commons Attribution Non Commercial (CC BY-NC 4.0) license, which permits others to distribute, remix, adapt, build upon this work non-commercially, and license their derivative works on different terms, provided the original work is properly cited, appropriate credit is given, any changes made indicated, and the use is non-commercial. See: http://creativecommons.org/licenses/by-nc/4.0/.

\section{ORCID iDs}

Carlos J Chaccour http://orcid.org/0000-0001-9812-050X

Cassidy Rist http://orcid.org/0000-0002-7558-8094

\section{REFERENCES}

1 World Health Organization. Global technical strategy for malaria 2016-2030. Geneva: WHO, 2015.

2 World Health Organization. World malaria report 2019. Geneva: WHO, 2019.

3 World Health Organization. High burden to high impact: a targeted malaria response. Geneva: WHO, 2018.

4 malERA Refresh Consultative Panel on Tools for Malaria Elimination. malERA: an updated research agenda for diagnostics, drugs, vaccines, and vector control in malaria elimination and eradication. PLoS Med 2017;14:e1002455.

5 Strode C, Donegan S, Garner P, et al. The impact of pyrethroid resistance on the efficacy of insecticide-treated bed nets against African anopheline mosquitoes: systematic review and metaanalysis. PLoS Med 2014;11:e1001619.

6 World Health Organization. The potential impact of health service disruptions on the burden of malaria: a modelling analysis for countries in sub-Saharan Africa. Geneva: WHO, 2020.

7 Killeen GF, Tatarsky A, Diabate A, et al. Developing an expanded vector control toolbox for malaria elimination. BMJ Glob Health 2017;2:e000211.

8 von Seidlein L, Peto TJ, Landier J, et al. The impact of targeted malaria elimination with mass drug administrations on falciparum malaria in Southeast Asia: a cluster randomised trial. PLoS Med 2019;16:e1002745.

9 World Health Organization. WHO preferred product characteristics: endectocide for malaria transmission control. Geneva: WHO, 2017.

10 Billingsley P, Binka F, Ivermectin R, The Ivermectin Roadmappers. A roadmap for the development of ivermectin as a complementary malaria vector control tool. Am J Trop Med Hyg 2020;102:3-24.

11 Meredith HR, Furuya-Kanamori L, Yakob L. Optimising systemic insecticide use to improve malaria control. BMJ Glob Health 2019;4:e001776.

12 Slater HC, Foy BD, Kobylinski K, et al. Ivermectin as a novel complementary malaria control tool to reduce incidence and prevalence: a modelling study. Lancet Infect Dis 2020;20:498-508.

13 Smit MR, Ochomo EO, Aljayyoussi G, et al. Safety and mosquitocidal efficacy of high-dose ivermectin when coadministered with dihydroartemisinin-piperaquine in Kenyan adults with uncomplicated malaria (IVERMAL): a randomised, double-blind, placebo-controlled trial. Lancet Infect Dis 2018;18:615-26.

14 Bockarie MJ, Hii JL, Alexander ND, et al. Mass treatment with ivermectin for filariasis control in Papua New Guinea: impact on mosquito survival. Med Vet Entomol 1999;13:120-3.

15 Slater HC, Walker PGT, Bousema T, et al. The potential impact of adding ivermectin to a mass treatment intervention to reduce malaria transmission: a modelling study. J Infect Dis 2014;210:1972-80.

16 Foy BD, Alout $\mathrm{H}$, Seaman JA, et al. Efficacy and risk of harms of repeat ivermectin mass drug administrations for control of malaria (RIMDAMAL): a cluster-randomised trial. Lancet 2019;393:1517-26.

17 Conteh L, Sicuri E, Manzi F, et al. The cost-effectiveness of intermittent preventive treatment for malaria in infants in subSaharan Africa. PLoS One 2010;5:e10313.

18 White MT, Conteh L, Cibulskis R, et al. Costs and cost-effectiveness of malaria control interventions--a systematic review. Malar J 2011;10:337.

19 Cirera L, Galatas B, Alonso S, et al. Moving towards malaria elimination in southern Mozambique: cost and cost-effectiveness of mass drug administration combined with intensified malaria control. PLoS One 2020;15:e0235631.

20 Griffin JT, Bhatt S, Sinka ME, et al. Potential for reduction of burden and local elimination of malaria by reducing Plasmodium falciparum malaria transmission: a mathematical modelling study. Lancet Infect Dis 2016;16:465-72.

21 Dev V, Sharma VP. The dominant mosquito vectors of human malaria in India. Anopheles mosquitoes-New insights into malaria vectors. IntechOpen, 2013.

22 Bank W. World development indicators. Washington, DC: The World Bank, 2018.

23 Conteh L, Shuford K, Agboraw E, et al. Costs and cost-effectiveness of malaria control interventions: a systematic literature review. Value Health 2021;24:1213-22. 
24 Keating J, Yukich JO, Mollenkopf S, et al. Lymphatic filariasis and onchocerciasis prevention, treatment, and control costs across diverse settings: a systematic review. Acta Trop 2014;135:86-95.

25 World Health Organization. World malaria report 2020. Geneva: WHO, 2020.

26 ClinicalTrials.gov. Repeat ivermectin mass drug administrations for control of malaria: a pilot safety and efficacy study (RIMDAMAL). Available: https://clinicaltrials.gov/ct2/show/study/NCT02509481 [Accessed 27 Dec 2020].

27 ClinicalTrials.gov. Mass drug administration of ivermectin and Dihydroartemisinin-piperaquine as an additional intervention for malaria elimination (MASSIV). Available: https://clinicaltrials.gov/ct2/ show/study/NCT03576313 [Accessed 27 Dec 2020].

28 ClinicalTrials.gov. Efficacy and safety of high-dose ivermectin for reducing malaria transmission: a dose finding study (IVERMAL).
Available: https://clinicaltrials.gov/ct2/show/study/NCT02511353 [Accessed 27 Dec 2020].

29 ClinicalTrials.gov. Adjunctive ivermectin mass drug administration for malaria control (MATAMAL). Available: https://clinicaltrials.gov/ct2/ show/NCT04844905 [Accessed 6 Aug 2021].

30 Kobylinski KC, Sylla M, Chapman PL, et al. Ivermectin mass drug administration to humans disrupts malaria parasite transmission in Senegalese villages. Am J Trop Med Hyg 2011;85:3-5.

31 Sylla M, Kobylinski KC, Gray M, et al. Mass drug administration of ivermectin in south-eastern Senegal reduces the survivorship of wild-caught, blood fed malaria vectors. Malar J 2010;9:1-10.

32 Patouillard E, Griffin J, Bhatt S, et al. Global investment targets for malaria control and elimination between 2016 and 2030. BMJ Glob Health 2017;2:e000176.

33 World Health Organization. Tailoring malaria interventions in the COVID-19 response. Geneva: WHO, 2020. 